\title{
Marketing e Islam: análisis de la Religión como nueva variable de microsegmentación en España
}

\author{
Pilar SÁNCHEZ GonZÁLEZ \\ ESIC Business \& Marketing School de Madrid \\ pilar.sanchez@esic.edu
}

\section{Resumen:}

El Marketing ya no se planifica desde una perspectiva nacional o internacional, sino "glocal", por un lado como estrategia inicialmente global pero, de forma inevitable, con adecuaciones locales. Ello implica microsegmentar las estrategias y plantear el análisis de nuevas variables. Si la Cultura ya había sido considerada como canalizadora de consumo, ahora la Religión, y en concreto el Islam, se está convirtiendo en una oportunidad de estudio. Este trabajo es el primer acercamiento en la búsqueda de características que relacionen la identidad musulmana con el Marketing y su consumo Halal.

Palabras clave: Identidad; Islam; Halal; microsegmentación cultural; perspectiva "glocal"

\section{Marketing and Islam: Religion analysis as a new variable microsegmentation in Spain}

\begin{abstract}
:
Marketing is no longer planning on a national or international level, but "glocal", firstly as overall strategy initially but, inevitably, with local adaptations. This implies micro segment strategies and raise new analysis variables. If Culture had been considered channelling consumption now Religion, specifically Islam, is becoming an opportunity to study. This work is the first approach in the search for features that Muslim identity in relation to Marketing and Halal consumption.
\end{abstract}

Key Words: Identity; Islam; Halal; microsegmentation cultural; "glocal” perspective

\section{Referencia normalizada:}

Sánchez González, P. (2014): Marketing e Islam: análisis de la religión como nueva variable de microsegmentación en España. Historia y Comunicación Social. Vol. 19. Núm. Especial Marzo. Págs. 117-127.

Sumario: 1. Introducción. 2. Marco metodológico. 3. Desarrollo. 4. Conclusiones. 5. Bibliografía.

\section{Introducción}

La realidad empresarial española está inmersa en un cambio estratégico desde dos perspectivas. Por un lado, y como consecuencia de los fenómenos migratorios en nuestro país, coexisten diversas etnias y religiones que han creado diferentes identidades culturales provocando, a su vez, interculturalidad en el mercado. Y por otro, debido 
a la crisis económica y a la tecnologización, las empresas realizan su planificación de Marketing buscando nuevos nichos de mercado internacional. En esta búsqueda constante la microsegmentación se afianza como estrategia de éxito. Además de las variables tradicionales (edad, sexo, clase socioeconómica, estilos de vida, etc.) el Marketing se plantea la "personalización" en sus campañas. Y ese proceso pasa por encontrar aquellos rasgos, especialmente definitorios, de los consumidores y de sus deseos de compra.

Creemos que una nueva variable a tener en cuenta, al hacer la planificación estratégica de Marketing, es la Religión. Los consumidores poseen vivencias que les hacen decidir. Dichas experiencias se afianzan en su vida creando perfiles de compra. El seguimiento de una religión u otra configura una identidad, definida por opciones permitidas, aconsejadas, prohibidas o anuladas. Y todas ellas trasmitidas desde una cultura determinada.

La Cultura de un pueblo pasa por la integración de las diferentes religiones en su estructura social. En el caso español conviven, aunque con diferencias notables de número de fieles, las tres religiones denominadas "del Libro": Católica, Islam y Judía.

Nuestro trabajo trata de analizar un marco conceptual que ayude a entender mejor el Islam, el consumo islámico y sus relaciones con la identidad cultural. Tendrá que proporcionar, asimismo, una serie de directrices y orientaciones que contribuyan a una planificación más eficiente del Marketing dirigido a este sector de consumidores.

Nos encontramos en las fases de revisión bibliográfica y en la elaboración de instrumentos teóricos contenidos en la misma. En concreto, esperamos dotarnos, a corto plazo, de algunas herramientas analíticas que nos ayuden a describir con más precisión el campo del consumo islámico en España.

La investigación, creemos, es importante desde dos perspectivas. Por un lado, histórica, porque España es un país con identidad musulmana y está imbricada en la sociedad. Y por otro lado, las empresas están ante la perspectiva "glocal", que supone dotarlas de una perspectiva global con adecuación local. En este caso, la consideración de identidad cultural y, por ende, religiosa es fundamental.

Tras una revisión de toda la literatura, se puede afirmar, que no hay en España aún, ningún estudio académico que relacione, desde esta perspectiva, Islam y Marketing.

\section{Marco metodológico}

El objetivo principal de este trabajo, que forma parte de la realización de una Tesis Doctoral, es analizar hasta qué punto la Religión, en este caso el Islam, influye en la toma de decisiones de Marketing. Desde dos perspectivas: la adecuación de la empresa para conseguir vender más, considerando la variable de la Religión como básica en los procesos de microsegmentación; y desde la alteridad como proceso 
imprescindible en las planificaciones estratégicas de las compañías que poseen un criterio de mercado claramente global y que, por tanto, necesitan una visión intercultural diferente.

Como objetivo subsidiario se pretende abrir nuevas líneas de investigación en un futuro inmediato creando, a su vez, un observatorio de rango académico que se encargue de estudiar, difundir y hasta predecir, comportamientos de una tipología de clientes muy específica: los musulmanes.

El método utilizado consta de una investigación sobre los principales autores que han estudiado y analizado la relación entre Cultura, Religión y Consumo. A partir de los resultados, realizaremos una investigación cuantitativa, por definir, para contrastar las hipótesis de partida. Tomaremos como punto de referencia lo que sucede en otros países e intentaremos adecuarlo al caso español desde los dos parámetros que consideramos básicos: desde empresas españolas hacia consumidores musulmanes españoles y desde multinacionales españolas hacia mercados musulmanes internacionales.

\section{Desarrollo}

Sobre la base de la búsqueda de una definición de cultura actual y partiendo de que para muchos autores, la cultura es "socialmente conservadora", preferimos recurrir a las palabras de Bauman (2013: 13), que decía así:

Bordieu con La distinción puso patas arriba el concepto original de "cultura" nacido con la ilustración y luego transmitido de generación en generación. El significado que describía, definía y documentaba Bordieu estaba a una distancia remota del concepto de "cultura" tal como se lo había modelado e introducido en el lenguaje corriente durante el tercer cuarto del siglo XVIII.

De acuerdo con su concepto original, la "cultura" no debía ser una preservación del statu quo sino un agente de cambio; más precisamente, un instrumento de navegación para guiar la evolución social hacía una condición humana universal.

Creemos, como Bordieu y Bauman, que este cambio constante configura una nueva realidad cultural. Es fruto de los movimientos migratorios y de la percepción de que el mundo se ha convertido en un mercado global. Hoy, la cultura no consiste en prohibiciones sino en ofertas. No consiste en normas, sino en propuestas. Tal como señaló Bordieu, la cultura se ocupa de ofrecer tentaciones y establecer atracciones, con seducción y señuelos en lugar de reglamentos, con relaciones públicas en lugar de supervisión policial: produciendo, sembrando y plantando nuevos deseos y necesidades en lugar de imponer el deber. Si hay algo en relación con lo cual la cultura de hoy cumple la función de un homeostato, no es la conservación del estado presente sino la abrumadora demanda de cambio constante. Podría decirse que sirve no tanto a las estratificaciones y divisiones de la sociedad, como al mercado de consumo orien- 
tado por la renovación de existencias. La nuestra es una sociedad de consumo: en ella, la cultura, al igual que el resto del mundo experimentado por los consumidores, se manifiesta como un depósito de bienes concebidos para el consumo (Bauman, 2013: 19).

Este nuevo concepto de cultura universal del consumo nos hace preguntarnos cuáles son las variables que tendrán que considerar los profesionales del Marketing a partir de ahora, puesto que la "búsqueda del futuro cliente" continúa demandando nuevos análisis, mucho más pormenorizados, de la realidad. Atrás quedaron segmentaciones con las variables básicas: edad, sexo, nivel de estudios y cultural, etc. Se impone la máxima personalización de las acciones. Si el cliente no las siente suyas, no compra el producto. La tecnología ha provocado que la competencia sea brutal. El cliente tiene la posibilidad de conseguir cualquier producto, de cualquier marca, procedente de cualquier país del mundo.

Es el momento del cambio. Ni los clientes, ni las empresas ni el mercado son iguales a hace muy pocos años. Los movimientos de población han provocado la mezcla multicultural. Las personas se desplazan de barrios, municipios, regiones, países y continentes buscando una nueva vida: "En une année, plus de 213 millions de personnes vivent durablemente sur une terre qui n'est pas celle d'oirgine" (United Nations. Departmente of Economic and Social Affairs, Population Division (Banon, 2012: 271).

En esa huida siempre llevan consigo dos conceptos: su "paladar", la forma de comer y de saborear es cultural, allá donde vayan identificarán sabores con emociones. Y también se llevarán su "alma"; su religión les ayudará a sobrevivir y a encontrarse con sus iguales. Para otra investigación queda analizar la simbología del "paladar". En este caso, nos vamos a centrar únicamente en el segundo concepto: la Religión.

La religión desempeña un papel muy importante en la cultura de la humanidad. Cultura y religión se influyen mutuamente. La religión da forma a los sistemas de creencias y prácticas que se inscriben en la cultura particular que le corresponda. La cultura, por su parte, influye sobre los modos de prácticas, la integración religiosa y las creencias necesarias para llevar a cabo sus rituales en la vida social. No hay cultura que actúe ajena a una religión, sea de una forma directa o indirecta. Y no hay cultura desarrollada sin el adecuado entendimiento de su religión (Parekh, 2005 y Padilla y Sánchez, 2013: 450).

La religión, en si misma, pretende aunar la realidad de la humanidad con la realidad universal. Por tanto, la profesión de unas creencias religiosas sirve para legitimar y conservar la realidad de una sociedad. Se convierte en catalizador y herramienta de transformación de la propia cultura de la sociedad que acoge, pudiendo contribuir a la cohesión del conjunto de la sociedad (Garreta, 2003). De hecho, la sociedad, la religión y su cultura están estrechamente interrelacionadas.

En el inicio de nuestra investigación queremos analizar, en primer lugar, la importancia que tiene el hecho religioso en la comunidad inmigrante dado que la mayo- 
ría de los musulmanes que lo son en España provienen de la inmigración. Nuestro trabajo se centra en la realidad española y en considerar la Religión como una variable más de microsegmentación. Por ello, creemos que es imprescindible el análisis de la relación religión-integración para poder diagnosticar pautas de consumo. En futuras investigaciones quedará el estudio de la religión como fenómeno de identidad en otros países.

Partimos de la teoría funcionalista de la religión, que otorga a ésta un importante papel en la vida e integración de las personas y pone de manifiesto las implicaciones sociales del hecho religioso (Bravo, 2012: 157). Esta teoría parte de cuatro realidades: contingencia, impotencia, escasez y frustración y privación: (i) Las contingencias e imprevistos en la vida de las personas causan inseguridad y desencanto. La crisis económica, la muerte, la enfermedad y en general los hechos negativos provocan desesperación e impotencia, en definitiva miedos. En estos casos la religión aporta significado a la vida. (ii) La religión crea una relación trascendental con un ser superior con el que no hay relación de igualdad y que genera sensación de impotencia. Pero también genera seguridad ante situaciones límite. Con motivo de movimientos migratorios las personas pierden sus referencias sociales y culturales, viven en otro país y con otros valores. La religión les aporta seguridad e identidad. (iii) La religión actúa como controladora social, legitima el orden establecido y crea mecanismos de control por lo que en ocasiones genera frustración. Así puede ser que inmigrantes en España puedan cuestionar la propia estructura social del país de acogida y generen una amenaza para la estabilidad social. Y (iv) la religión tiene un importante papel en la formación de la identidad individual y colectiva de las personas. En el caso de inmigrantes cumple funciones puramente sociales además de religiosas. En el lugar de culto encuentran paz y sosiego en momentos de dificultad pero también reciben consejo sobre las cuestiones de la vida y se encuentran con personas de su lugar de origen. Por todo ello, la religión adquiere el concepto de "apoyo de vida". A mayor dificultad de integración mayor necesidad de búsqueda de recursos individuales para sobrevivir.

Como señala Bravo (2012: 158): para la población inmigrante, la religión cumple unas funciones sociales distintas de las puramente religiosas, aunque éstas sigan siendo las más importantes, que inciden positivamente en su proceso de integración. En el lugar de culto encuentran paz y sosiego en momentos de dificultad consecuencia del tránsito migratorio, reciben consejo sobre las cuestiones de la vida y encuentran y se reúnen con personas de su tierra. Por todo ello, la religión adquiere un mayor protagonismo en la vida de las personas cuando éstas se encuentran en una situación de minoría social y es que cuánto más difícil sea la integración de estas personas en la nueva sociedad más importante es la obtención de estima y autoestima a partir de recursos propios.

En muchas ocasiones, el consumo de productos identificados con el grupo de pertenencia les ayuda a sobrellevar la situación de minoría y a mejorar la identidad con su consumo. Éste es el principio del que partimos en nuestra investigación. La compra de los clientes se ve influida por su forma de sentir su religión. La frase de 
Sánchez (2011), compras como rezas, enmarcada en sus primeros análisis de la relación Islam y Marketing, supone mucho más de lo que podría ser un eslogan comercial. Pensamos que representa una identidad simbiótica entre la persona y su alma, y que le hace comportarse, en los momentos de toma de decisiones comerciales, de una forma específica y diferenciada.

Desde esta perspectiva de estudio nos proponemos analizar la situación de la religión en España. En este momento, el Estado español clasifica las confesiones religiosas en cuatro grupos. En el primer grupo se hallaría la Iglesia Católica, ya que es la única que tiene acuerdos de carácter internacional. En un segundo grupo estarían las que han firmado acuerdos con el Estado, como son las religiones musulmana, judía y evangélica (o protestante). En un tercer grupo se encuentran las religiones denominadas de "notorio arraigo", como las de los mormones, los ortodoxos y los testigos de Jehová. Por último, existen otras religiones con menor representación en España, como podrían ser los odinistas o la iglesia de la cienciología, entre otras.

A todas estas religiones les afecta, por supuesto, el Art. 16 de la Constitución de 1978, en el que se consagra el principio de libertad religiosa y de culto, así como la Ley Orgánica 7/1980, de 5 de Julio, de libertad religiosa que desarrolla el mencionado artículo. De esta ley de libertad religiosa debe destacarse que el artículo $2 b$ ) reconoce el derecho de las Iglesias, Confesiones y Comunidades religiosas a conmemorar sus festividades, celebrar sus ritos matrimoniales, recibir una sepultura digna y recibir enseñanza religiosa (Vela y Ballesteros, 2011: 367).

En este trabajo se ha tomado como referencia una de las tres religiones del Libro, el Islam, por varios motivos: evidentemente, por la cercanía en cuanto a raíces culturales; España, históricamente, ha sido tierra habitada por judíos, moros y cristianos, y la historia y el patrimonio cultural del país están plagados de referencias mestizas-mozárabes, sefardíes y mudéjares, que hablan de esa mezcla de culturas. Juderías y morerías son habituales en muchas ciudades y pueblos de España y el mismo lenguaje castellano está cargado de términos provenientes de dichas culturas (Vela y Ballesteros, 2011: 362).

Un segundo motivo es que es una de las religiones más claras y precisas en cuanto a dogmas y normas que influyen en la forma de vida de sus creyentes. Por último, un tercer motivo, es que la crisis económica, que también afecta a España, está provocando que muchas de nuestras empresas se vean en la necesidad de buscar nuevos nichos de mercado mucho más específicos, microsegmentados y que se planteen la posibilidad de convertirse en multinacionales que tienen que dirigir sus estrategias a otros mercados de países con los que mantenemos relaciones empresariales, bien por proximidad física y cultural: Marruecos, Túnez, Arabia Saudí. O bien porque, en estos momentos, son países con una riqueza energética y económica de tal magnitud que son lo suficientemente interesantes para intentar posicionarnos en ellos. Nos referimos, por ejemplo, a Qatar o Malasia, por ejemplo. Todos ellos son países que, aunque de diferentes formas, profesan el Islam. Las empresas españolas deberían 
conocer sus preceptos básicos para relacionarse mejor con ellos ya que constituyen, según nuestro criterio, una alternativa económica nada desdeñable.

Desde esta percepción, el Islam tiene un papel fundamental en la cultura de la comunidad islámica, ya que las personas que la profesan consideran la religión como elemento esencial en sus vidas. Creen que dicha religión y sus creencias son elementos que les ayudan a conservar su propia cultura (Sánchez, 2012: 22). Su evolución depende de cómo se interrelacionan los elementos que la componen: idiomas, costumbres o expresiones artísticas y, por otro lado, de cómo se interpretan los textos que siguen.

Tal como sostienen los islamólogos Mohamed Bahige Mullá y Abdelouahab Atta (Padilla y Sánchez, 2013: 450-451), el Islam consta de tres elementos: (i) Fe y culto: son particularidades personales que ocupan el espacio más restringido. El culto mismo se divide en culto a Dios en reconocimiento a Su Unicidad (oraciones y peregrinación) y culto a Dios (Ortopraxia), que es todo acto cívico realizado al servicio de la sociedad para agradar a Dios. El Islam es, ante todo, una religión social cuyo objetivo primordial es preparar al ser humano para desenvolverse sanamente en la vida de este mundo. (ii) El Derecho o Sharia: es el conjunto de disposiciones legales; muy parecidas a lo que llamamos ley marco y por tanto, abierto a incluir innovaciones contextuales de tipo legislativo que regulan la vida pública. Siempre y cuando no violen la base y el espíritu del Islam. A nivel colectivo, tiene valor normativo sólo en un país que la adopte como ley vigente y, por lo tanto, no nos concierne en España. La Sharia "tiene como particularidad el saber adaptarse a distintas situaciones gracias a la ausencia de una autoridad única que controla la ortodoxia" (Necco, 2010). Y (iii) el Mensaje es el tercer elemento básico. El conjunto de valores normativos que no difieren mucho de la escala de valores universales y que tiende a establecer una base sólida para el desarrollo de la sociedad humana.

El Mensaje islámico inculca los siguientes elementos: (i) La variedad de credos es una voluntad divina (Corán 10/99 y 18/29) y en consecuencia, la compulsión en materia de fe es un delito (pecado) mayor. (ii) La libertad de creer y de expresar la cultura religiosa es un valor moral y un derecho inalienable y, en virtud de este valor, los lugares de culto, los clérigos y el patrimonio religiosos gozan de inmunidad legal. (iii) El desprecio dirigido contra los émbolos de cualquier religión (blasfemia) es un delito punible (Corán 6/106-108). (iv) Conocer al "diferente" es un deber cívico, porque el "diferente" es el prójimo que siempre tendrá algo que comunicar y para conocerle bien hay que seguir la vía del diálogo y de la dialéctica (Corán 49/13 y 34/24). (v) La construcción social es el campo donde tenemos que competir y concurrir, de tal manera que la personalidad social del individuo se consolide con la personalidad moral (Corán 2/48). (vi) La convivencia intercomunitaria es una necesidad para el desarrollo social integral. Todos los seres humanos son miembros de una sola familia universal, el más preciado por Dios de entre ellos es el que mejor sirve a la Familia. Por tanto, el objetivo del Islam es dotar a sus creyentes de la herramienta necesaria para que goce de una vida sana en consonancia con el ecosistema en el que habite. 
Una vez analizados brevemente los principios del Islam, veamos cómo se define la realidad de los musulmanes en nuestro país. Padilla y Sánchez (2013: 453) analizan la comunidad musulmana y detallan que en el mundo se compone, aproximadamente, de mil millones de personas. Dieciséis millones de musulmanes viven en Europa (Roy, 2012) y aproximadamente, dos millones en España. De estas cifras cabe señalar que el $70 \%$ de las personas que se declaran musulmanes en España, son inmigrantes. Destaca el colectivo marroquí como grupo más numeroso, seguido, en este orden, por argelinos, paquistaníes, iraníes, libaneses, sirios y tunecinos, entre otras nacionalidades. El 30\% restante son españoles, que según Planet (2008), incluye a inmigrantes musulmanes que han obtenido la nacionalidad española y a españoles que se han convertido al culto islámico.

Para Losada (1995: 189-199), se pueden diferenciar cuatro corrientes del Islam en España y son las siguientes: (i) Practicantes instalados: Son los inmigrantes de la primera generación que, en general, intentan evitar asimilarse a la sociedad de acogida. (ii) Musulmanes de segunda generación: Aquellas personas que no rechazan la cultura ni la religión de sus padres y se adaptan al modo de vida occidental, buscando una adecuada integración en la sociedad de acogida. (iii) Musulmanes islamistas: Con una percepción negativa en cuanto a la emigración de su país, debido al miedo a la asimilación y a la pérdida de su identidad religiosa. (iv) Musulmanes sociológicos: Con una referencia al Islam cultural más que al Islam de culto.

Así pues, la situación del culto islámico en el territorio español, como en casi todos los países europeos, está representado por la llegada de inmigrantes procedentes de países musulmanes, y por la existencia de un marco jurídico e institucional que regula las cuestiones religiosas y la presencia del culto islámico (Planet, 2008: 1-32).

A pesar de tener legislación, en España y en Europa, no se supo promover la diversidad religiosa. Esto permitió una influencia extranjera considerable en los ámbitos de financiación de los grupos radicales, construcción de espacios religiosos o la enseñanza del Islam. Todo ello ha perjudicado el proceso de integración del colectivo musulmán, autóctono e inmigrante, aunque deja margen a la producción de un Islam español que aboga por le respeto y la compatibilidad de los valores españoles y la concordia.

En esa línea de convivencia y de respeto por el otro, nos encontramos. Por tanto, podemos considerar que en España, se ha configurado un nuevo microsegmento de Marketing: el cliente musulmán. Con identidad suficiente desde las nuevas perspectivas de Marketing, basadas en la personalización y el " 1 to 1". Desde este punto de vista estratégico, creemos que comienza un nuevo reto marketiniano, al considerar la religión, en este caso el Islam, como variable de segmentación.

Abiertas quedan las próximas líneas de investigación: la realidad de las empresas españolas en países musulmanes y el análisis del consumidor musulmán global. 


\section{Conclusiones}

Una vez analizada la relación Cultura, Religión y Consumo, en nuestro caso el Islam, llegamos a la conclusión de que existe una vinculación directa entre ellas. La cultura de un pueblo a través de sus religiones genera unos consumidores con características determinadas. A mayor presencia de una religión, sea cual fuere, en la vida de los ciudadanos de un país, mayor capacidad de microsegmentación en las estrategias de Marketing. Creemos que la tendencia es la adaptación paulatina de la frase "compras como rezas". Dicha frase será refutada con la investigación que estamos preparando para la realización de la Tesis Doctoral.

En el caso de las empresas españolas, creemos que supone una oportunidad de negocio optar por el Islam como religión elegida para la microsegmentación. Por un lado por la vertiente histórica característica de nuestro país y por otro lado por la proximidad o vecindad de los países limítrofes que configuran una nueva tipología de mercado, por ejemplo, el "mercado mediterráneo", con características de consumo afines y con relaciones internacionales históricas que nos benefician en nuestro entramado empresarial.

Estudiar el Islam como posible variable en las estrategias de Marketing puede parecer arriesgado. Sin embargo, creemos que supone la adecuación de un proceso imparable desarrollado en el ámbito internacional. Hace ya unos cuantos años que en otros países se trabaja el concepto Islamic Marketing, como un tipo más de diversificación empresarial. Autores como Nestorovic (2010) estudian la adecuación de los pilares tradicionales del Marketing, las $4 \mathrm{p}$ de Kotler: product, price, place and promotion y las convierten para el mercado musulmán en las 4 f: faith, food, finance and fashion. Pensamos, con humildad, que este puede ser el camino de la integración. Las $4 \mathrm{f}$ representan la máxima adecuación a su nueva realidad de vida y por tanto del Marketing. La Fe, como pilar fundamental que marca el consumo. La Comida porque representa todo un proceso de integración en la forma de vida. Las Finanzas porque acaban de alcanzar el reconocimiento internacional de eficacia monetaria y porque suponen una forma de economía saneada frente a la banca tradicional y, por ende, con un futuro prometedor. Y, por último, la Moda, como máxima expresión de externalizar la forma de rezar, en definitiva, el "alma".

Todas ellas están siendo tratadas en la investigación académica que estamos realizando. $Y$, aunque sin datos definitivos, podemos augurar que van a ser muy reveladores. 


\section{Bibliografía}

ABUMALHAM, M. (1995). Comunidades islámicas en Europa. Madrid: Trotta.

BANON, P. (2012). "Globalisation des cultures, quelle novelle éthique pour l'enterprise?" En BARTH, I. (2012). Management et religions, décryptage d'un lien indefectible. París:EMS.

BAUMAN, Z. (2013). La cultura en el mundo de la modernidad líquida. Madrid: Fondo de Cultura Económica.

BRAVO, L. (2012). "Las comunidades religiosas como espacios de acogida e integración de los inmigrantes: especial atención a las iglesias evangélicas étnicas, La integración aislada". En TSHITSHI NDOUBA, K. (2012). Inmigración y comunidades religiosas, implicaciones para las políticas de integración de los inmigrantes. Madrid: Instituto de Estudiso Jurídicos Internacionales.

LOSADA, T. (1995). "Inmigración musulmana: restos humanos, culturales y religiosos". En NECCO, E. (2010). Argumentos de bioética en el Islam: Aborto, planificación familiar e inseminación artificial. Madrid: CantArabia.

NESTOROVIC, C. (2010). Marketing Islámico. Milán: Egea.

PAREKH, B. (2005). Repensando el multiculturalismo. Madrid: Istmo.

PLANET, A. (2008). "Islam y emigración: elementos para un análisis y propuestas de gestión”. En (2008). Foro de Inmigración. Madrid: Centro de Estudios Políticos y Constitucionales, p. 1-32.

ROY, O. (2012). "Los nuevos islamistas". En Foreign Policy, abril 2012. Madrid: Washingtonpost Newsweek Interactive. Disponible en:

http://www.revistas.ucm.es/index.php/ESMP/article/download/42052/40033 [19-092013].

SANCHEZ, P. (2012). "Londres 2012: Imaginemos entre culturas". En Revista de Comunicación, $\mathrm{n}^{\mathrm{o}}$ 25, España: Wolters Kluwer, p. 21-23. Disponible en: http:// revistadecomunicacion.wke.es/noticias_base/fallo_acceso/imaginemos-entre-culturas [19-09-2013].

SÁNCHEZ, P. y PADILLA, G. (2013). "La importancia del tratamiento de la religión en medios de comunicación. El caso del Islam en España”. En Estudios sobre el Mensaje Periodístico, $\mathrm{n}^{\mathrm{o}} 19$.

VELA, C. y BALLESTEROS, C. (2011). "La influencia de las creencias religiosas en el consumo. Una aproximación desde las tres religiones". En Revista de las Facultades de Derecho y Ciencias Económicas y Empresariales. Madrid: ICADE. p. 361-392. 


\section{La autora}

Pilar Sánchez González. Actualmente, Profesora del Departamento de Humanidades de ESIC. Realizando la Tesis Doctoral en la Universidad Rey Juan Carlos de Madrid. Licenciada en Sociología y en Ciencias Políticas. Master en Marketing Directo y Relacional por ESIC. Personal docente e investigador de ESIC desde 1993: profesora de Estructura Social, Ética Empresarial, Historia de las Instituciones Públicas y Marketing Directo y Relacional. Colabora periódicamente con universidades internacionales: ESAN en Lima, Perú; Fachhochschule en Dortmund, Alemania; y ESC, en Pau, Francia. Compagina la carrera académica con la Asesoría Profesional, y desde 1997, es directora de Gamón Pumareta Asesores en Marketing en Madrid. 